\title{
Imaging in the aftermath of COVID-19: what to expect
}

\author{
Lukas Ebner $^{1}$ (D) Manuela Funke-Chambour ${ }^{2} \cdot$ Christophe von Garnier $^{3} \cdot$ Gilbert Ferretti $^{4} \cdot$ Benoit Ghaye $^{5}$. \\ Catherine Beigelman-Aubry ${ }^{6}$
}

Received: 29 September 2020 / Revised: 18 October 2020 / Accepted: 4 November 2020 / Published online: 28 December 2020

(C) European Society of Radiology 2020

\section{Dear Editors}

During the past six months, the COVID-19 pandemic has profoundly altered society and affected health care systems on a global scale. After initial reports of a new form of pneumonia in early 2020 , the situation rapidly evolved into a worldwide phenomenon. The early experience with COVID-19 and its severe respiratory symptoms reinforce the role of radiology in the diagnosis and monitoring of patients. Since then, a plethora of reports and scientific studies focused on the critical role of imaging in the triage and diagnosis of patients [1]. We initially learned that the COVID-19 pneumonia does present with characteristic imaging findings in the acute stage $[2,3]$. Although not specific, these findings led to the broad use of either CT imaging or chest radiographs for the assessment of COVID-19. This was of particular interest since numerous studies observed increased false negative rates for RT-PCR and abnormal CT findings were detectable in otherwise asymptomatic individuals.

Since February 2020 with the first cases of the novel COVID-19 reported, the social, economic and particularly medical landscape has widely adapted to the new situation.

Lukas Ebner

lukas.ebner@insel.ch

1 Department of Diagnostic, Interventional and Paediatric Radiology, Inselspital, Bern University Hospital, University of Bern, Bern, Switzerland

2 Department of Pulmonary Medicine, Inselspital, Bern University Hospital, University of Bern, Bern, Switzerland

3 Division of Pulmonary Medicine, Department of Medicine, Lausanne University Hospital (CHUV), University of Lausanne, Lausanne, Switzerland

4 Service de radiologie diagnostique et interventionnelle, $\mathrm{CHU}$ Grenoble Alpes, Grenoble, France

5 Department of Radiology, Cliniques universitaires Saint Luc, Brussels, Belgium

6 Radiodiagnostic and Interventional Radiology department, University of Lausanne, Lausanne, Switzerland
In many countries, the so-called first wave of infections has diminished, but a gradual re-increase of infections can be expected for fall and winter. Simultaneously, the first patients that recovered from COVID-19 in early 2020 are now presenting with sequelae from the initial Sars-CoV-2 infection.

We are presently facing patients that recovered from acute COVID-19 pneumonia, some with ARDS, and that were discharged having recovered from the acute illness. Nevertheless, some of these patients report ongoing dyspnoea and desaturate on exercise.

In the aftermath of a COVID-19 pneumonia, we observe in some patients with respiratory symptoms a suggestive pattern on CT images not previously reported in the context of COVID-19. In a subgroup of cured patients, we encounter a multifocal and/or anterior predominant pattern of hypoattenuated areas in the lung parenchyma 3 months after discharge. These areas frequently involve several adjacent, secondary lobules that are sharply demarcated from the surrounding lung parenchyma. In some cases, ground glass bands circumscribing these hypoattenuated areas may be observed. This suspected geographic hyperinflation of pulmonary lobules is characterised by markedly decreased lung density. Additionally, in some cases, the perilobular septae are bulging towards the periphery, reinforcing the detectability and the authenticity of this pattern on inspiratory CT scans. This air trapping could also be confirmed on expiratory scans (Fig. 1), but we believe that the distinct morphology and significant decrease intensity may not require systematic use of expiratory CT scan protocols. In our experience, the identification of hyperinflated lobules inspiratory CT scans is facilitated by employing narrow window settings and minimal intensity projections on a series reconstructed with thin slices and soft kernel (Figs. 1 and 2).

These CT findings are suggestive of small airways disease (SAD). In patients who initially suffered from ARDS, additional hallmark findings are reticulations, architectural distortions with bronchiectasis and curvilinear lines, as well as honeycombing in some cases. These CT features are attributable to sequelae of alveolar damage/ARDS and therefore less 


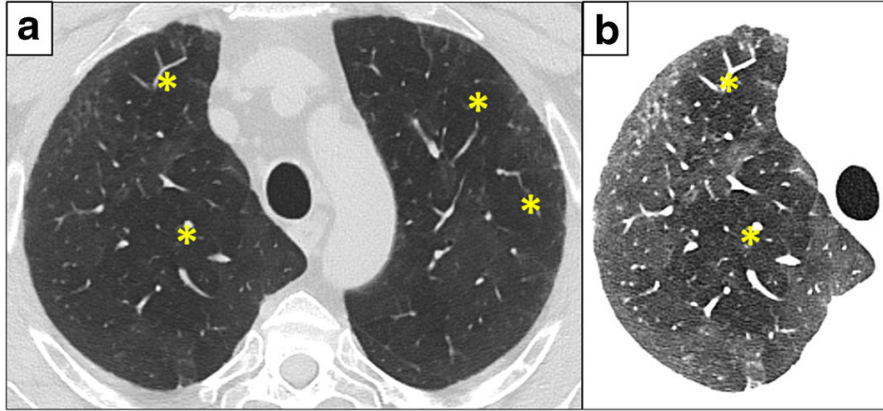

Fig. 1 Three-month follow-up CT scan of a patient after COVID-19 pneumonia presenting with exertional dyspnoea. Sharply demarcated areas of hypoattenuating lung parenchyma are depicted in both upper lobes (a). Reading the same acquisition on inspiration with narrow window settings on a series reconstructed in thin slices with soft kernel
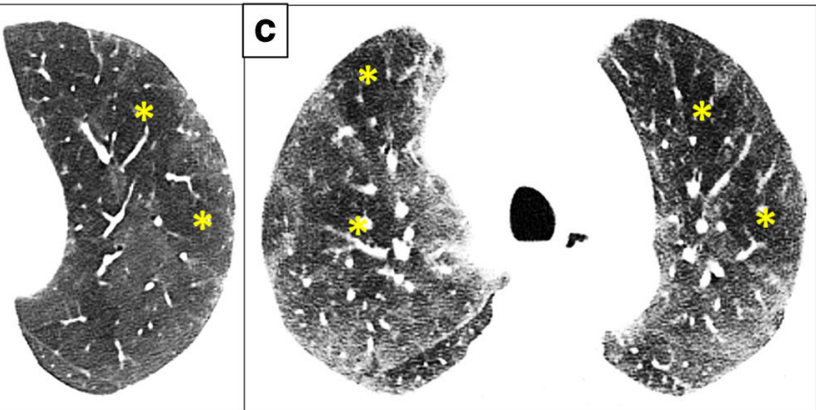

facilitates the detection of these abnormal areas and enforces a mosaiclike attenuation pattern (b). An additionally performed expiratory scan confirms the SAD owing to air trapping that matches with several hypoattenuating areas detected in (c). Note the predominant hypoattenuation pattern in this case characteristic of COVID-19 per se. When added together, postCOVID-19 CT scans may resemble the so-called head-cheese sign described in chronic hypersensitivity pneumonitis, a disorder that primarily affects the peribronchial area.

In the follow-up of symptomatic post-COVID-19 patients, the reported signs are pivotal for the understanding of pathological mechanisms involved with SARS-CoV-2 infection. Based on the $\mathrm{CT}$ patterns we encountered in cured COVID-19 patients, we noticed that some patients display a SAD predominance, while others present a predominance of classical fibrotic changes or a mixed type pattern. Of note, SAD was also reported in previous MERS, SARS and severe

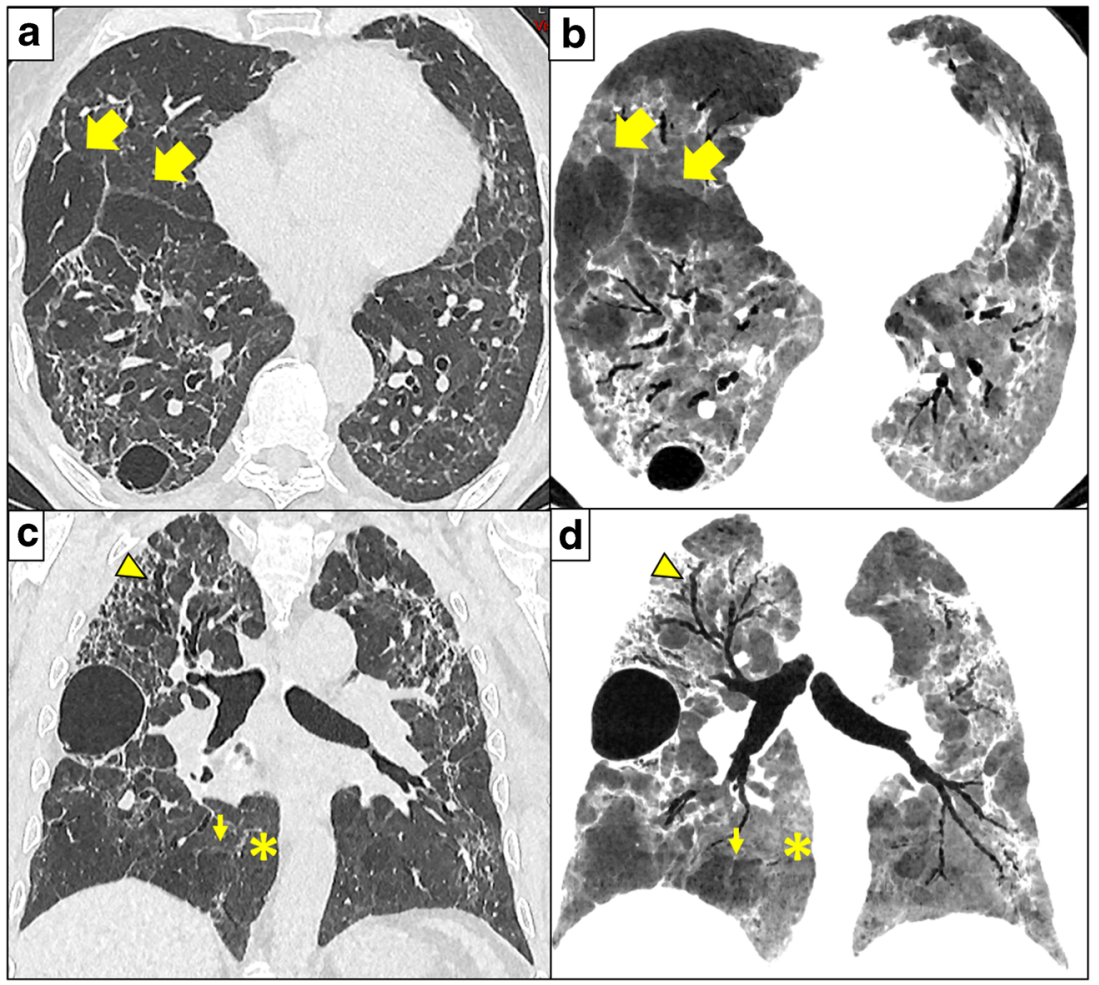

Fig. 2 Three months after COVID-19 and ARDS, CT shows severe architectural distortion and traction bronchiectasis (arrowhead in $\mathbf{c}$ and d). These findings are frequent in patients that recovered from ARDS. However, multi-lobular hypoattenuation pattern is also present (arrows) on axial 1-mm-thick slices reconstructed with lung kernel (a) and 10-mmthick minimum intensity projections (mIP) slices with soft kernel (b), and on coronal reformats 1-mm-thick reconstructed with lung kernel (c) and 10-mm-thick minimum intensity projections with soft kernel (d). The
mIP post-processing (b, d) highlights a geographic, lobular hypoattenuation pattern that most likely represents multifocal small airways disease. Presumably hyperinflated lobules further exhibit convex perilobular septae towards normal lung parenchyma (small arrows) that should correspond to an air trapping on expiration. Note that the normal lung parenchyma (stars) is more easily assessed on mIP reformats with narrow window settings $(\mathbf{b}, \mathbf{d})$, than on classical lung analysis $(\mathbf{a}, \mathbf{c})$. Note pneumatoceles in subpleural location described in COVID-19 pneumonia 
influenza infection and ARDS [4-6]. A limiting factor of our findings is that not all patients initially received a baseline CT and we can therefore not completely rule out preexisting conditions such as SAD.

These preliminary findings highlight the importance of imaging not only in the acute setting but also in the followup of COVID-19. As we adapt to the pandemic, the death toll is declining and hopefully mild to moderate infections will continue to surpass severe cases. Nevertheless, health care providers will face patients afflicted with long-term impairment. We suggest to scrutinise post-COVID-19 CT scans for findings of mosaic pattern, likely representing air trapping, and indicative of a potential distal airway obstruction. Future research should further address the complex association of SAD and reported vascular involvement [7, 8]. It has to be determined if and to what degree microvascular thrombosis may contribute to bronchiolar constriction or SAD [9].

Another critical aspect is the possible discrepancy between imaging findings and the clinical presentation of patients. To date, this relationship is still unclear. While some patients with minimal findings on chest CT may show a severe decline in lung function, there are patients with extensive diseases that exhibit relatively normal lung function. A correlation between imaging findings and moderate to severe disease course was identified on initial presentation [10], but focused and welldesigned studies are needed to explore lung function loss and dynamic CT findings in mid- to long-term follow-up of COVID-19.

In the light of increasing numbers of patients suffering late effects of COVID-19, we emphasise the importance of longitudinal evaluation to assess potential reversibility of the observed CT patterns.

Funding The authors state that this work has not received any funding.

\section{Compliance with ethical standards}

Guarantor The scientific guarantor of this publication is C. BeigelmanAubry.

Conflict of interest The authors of this manuscript declare no relationships with any companies, whose products or services may be related to the subject matter of the article.
Statistics and biometry No complex statistical methods were necessary for this paper.

Informed consent Written informed consent was not required for this study because of editorial purpose.

Ethical approval Institutional Review Board approval was not required.

Methodology

- Editorial comment

\section{References}

1. Revel M, Parkar AP, Prosch H et al (2020) COVID-19 patients and the radiology department - advice from the European Society of Radiology (ESR) and the European Society of Thoracic Imaging (ESTI). Eur Radiol 30:4903-4909

2. Zhou Z, Guo D, Li C et al (2020) Coronavirus disease 2019: initial chest CT findings. Eur Radiol 30:4398-4406

3. Wang H, Wei R, Rao G, Zhu J, Song B (2020) Characteristic CT findings distinguishing 2019 novel coronavirus disease (COVID19) from influenza pneumonia. Eur Radiol 30:4910-4917

4. Ketai L, Paul NS, Wong KT (2006) Radiology of severe acute respiratory syndrome (SARS). The emerging pathologicradiologic correlates of an emerging disease. J Thorac Imaging 21:276-283

5. Hosseiny M, Kooraki S, Gholamrezanezhad A, Reddy S, Myers L (2020) Radiology perspective of coronavirus disease 2019 (COVID-19): lessons from severe acute respiratory syndrome and Middle East respiratory syndrome. AJR Am J Roentgenol 214:1-5

6. Luyt CE, Combes A, Becquemin MH et al (2012) Long-term outcomes of pandemic 2009 influenza a(H1N1)-associated severe ARDS. Chest 142(3):583-592

7. Aiolfi A, Bruni B, Biraghi T et al (2020) Late histological findings in symptomatic COVID-19 patients. Medicine 28:e21046

8. von der Thüsen J, van der Eerden M (2020) Histopathology and genetic susceptibility in COVID-19 pneumonia. Eur J Clin Invest 50:e13259

9. Patel BV, Arachchillage DJ, Ridge CA et al (2020) Pulmonary angiopathy in severe COVID-19: physiologic, imaging, and hematologic observations. Am J Respir Crit Care Med 202:690-699

10. Fang X, Li X, Bian Y, Ji X, Lu J (2020) Relationship between clinical types and radiological subgroups defined by latent class analysis in 2019 novel coronavirus pneumonia caused by SARSCoV-2. Eur Radiol 30:6139-6150

Publisher's note Springer Nature remains neutral with regard to jurisdictional claims in published maps and institutional affiliations. 